\title{
RIABOUCHINSKY FLOWS IN MAGNETOHYDRODYNAMICS
}

\author{
BY \\ O. P. CHANDNA AND F. LABROPULU \\ University of Windsor, Windsor, Ontario, Canada
}

1. Introduction. This work is devoted to an investigation of exact solutions for steady plane flows of a viscous incompressible and electrically conducting fluid in the presence of a magnetic field by following a fluid dynamic technique of using compatibility or integrability equations for the flows. Ratip Berker [1,2] has used and thoroughly documented the application of integrability equations for incompressible and compressible non-MHD fluid flows. We study flows where the streamfunction defined by the continuity equation is assumed to be linear with respect to $y$ as an application of this technique. Flow problems involving such a form of the streamfunction are called Riabouchinsky type problems.

Riabouchinsky [3] extended flows for which the streamfunction $\psi(x, y)$ was of the form $\psi(x, y)=y F(x)$. Ratip Berker [1] generalized Riabouchinsky's work by considering flows where $\psi(x, y)=y F(x)+G(x)$. Kaloni and Huschilt [4] obtained solutions to these problems for non-Newtonian fluids.

We examine three types of magnetohydrodynamic (MHD) flows in this paper. These are aligned MHD, transverse MHD, and constantly-inclined MHD flows. We consider fluids having finite or infinite electrical conductivity. Since electrical conductivity is finite for most liquid metals, our accounting for finite electrical conductivity makes the flow problems realistic and attractive from both a mathematical and a physical point of view. We also include electrically conducting fluids of infinite electrical conductivity to make a thorough study of some of these fluid flows and to recognize the dawn of superconductivity in science.

The plan of this paper is as follows. In Sec. 2, the governing equations are written in a suitable form by using the definitions of aligned, transverse and constantlyinclined MHD flows. In Sec. 3, the compatibility or integrability equations for these flows are derived. In Secs. 4-6, we obtain solutions to Riabouchinsky type problems for aligned, transverse, and constantly-inclined flows. Motivation for carrying out this research is given in Sec. 7.

2. Flow equations. The steady flow of a viscous, incompressible, and electrically conducting fluid, in the presence of a magnetic field, is governed by the system of 
seven equations [5]:

$$
\begin{gathered}
\operatorname{div} \mathbf{V}=0, \\
\rho(\mathbf{V} \cdot \operatorname{grad}) \mathbf{V}+\operatorname{grad} p=\mu^{*}(\operatorname{curl} \mathbf{H}) \times \mathbf{H}+\mu \nabla^{2} \mathbf{V}, \\
\operatorname{curl}(\mathbf{V} \times \mathbf{H})-\frac{1}{\sigma \mu^{*}} \operatorname{curl}(\operatorname{curl} \mathbf{H})=\mathbf{0},
\end{gathered}
$$

where $\mathbf{V}, \mathbf{H}, p, \rho, \mu, \mu^{*}$, and $\sigma$ are, respectively, the velocity field vector, the magnetic field vector, the fluid pressure function, the constant fluid density, the constant coefficient of viscosity, the constant magnetic permeability, and the constant electrical conductivity. The magnetic field $\mathbf{H}$ satisfies an additional condition expressing the absence of magnetic poles in the flow given by

$$
\operatorname{div} \mathbf{H}=0 .
$$

Plane aligned flows. For these flows, the magnetic field vector is everywhere parallel to the velocity field and all the flow variables are functions of the rectangular coordinates $x$ and $y$ only. Employing this definition, we have $\mathbf{H}=f(x, y) \mathbf{V}$, where $f(x, y)$ is some scalar function, and Eqs. (1)-(4) take the form

$$
\begin{gathered}
\frac{\partial u}{\partial x}+\frac{\partial v}{\partial y}=0 \quad \text { (continuity) } \\
\rho\left[u \frac{\partial u}{\partial x}+v \frac{\partial u}{\partial y}\right]+\frac{\partial p}{\partial x}=\mu \nabla^{2} u-\mu^{*} f\left(u^{2}+v^{2}\right) \frac{\partial f}{\partial x}-\mu^{*} f^{2} v\left[\frac{\partial v}{\partial x}-\frac{\partial u}{\partial y}\right] \\
\rho\left[\frac{\partial v}{\partial x}+v \frac{\partial v}{\partial y}\right]+\frac{\partial p}{\partial y}=\mu \nabla^{2} v-\mu^{*} f\left(u^{2}+v^{2}\right) \frac{\partial f}{\partial y} \\
+\mu^{*} f^{2} u\left[\frac{\partial v}{\partial x}-\frac{\partial u}{\partial y}\right] \quad \text { (linear momentum) } \\
\frac{\partial}{\partial x}(f v)-\frac{\partial}{\partial y}(f u)=k, \quad \nu_{H}=\frac{1}{\mu^{*} \sigma} \neq 0 \quad \text { (diffusion), } \\
u \frac{\partial f}{\partial x}+v \frac{\partial f}{\partial y}=0 \quad \text { (solenoidal), } \\
\mathbf{H}=f \mathbf{V} \quad \text { (aligned), }
\end{gathered}
$$

where $k$ is an arbitrary constant of integration obtained by integrating the vector diffusion equation (3). The diffusion equation for aligned flows is identically satisfied when the fluid is infinitely electrically conducting so that $\nu_{H}=1 /\left(\mu^{*} \sigma\right) \rightarrow 0$. However, for finitely conducting fluids, the functions $f, u$, and $v$ must satisfy the additional equations (8).

Plane transverse flows. For plane transverse flows, the magnetic field vector is everywhere normal to the flow plane and all the flow variables are functions of the rectangular coordinates $x$ and $y$ only. Employing $\mathbf{V}=(u, v, 0)$ and $\mathbf{H}=(0,0, H)$ 
in Eqs. (1)-(4), these flows are governed by the system of four equations:

$$
\begin{gathered}
\frac{\partial u}{\partial x}+\frac{\partial v}{\partial y}=0 \quad \text { (continuity) } \\
\rho\left(u \frac{\partial u}{\partial x}+v \frac{\partial u}{\partial y}\right)+\frac{\partial}{\partial x}\left(p+\frac{1}{2} \mu^{*} H^{2}\right)=\mu \nabla^{2} u \quad \text { (linear momentum) } \\
\rho\left(u \frac{\partial v}{\partial x}+v \frac{\partial v}{\partial y}\right)+\frac{\partial}{\partial y}\left(p+\frac{1}{2} \mu^{*} H^{2}\right)=\mu \nabla^{2} v \\
u \frac{\partial H}{\partial x}+v \frac{\partial H}{\partial y}-\nu_{H} \nabla^{2} H=0 \quad \text { (diffusion) }
\end{gathered}
$$

in four unknown functions $u(x, y), v(x, y), p(x, y)$, and $H(x, y)$. Here $\nu_{H}=$ $1 /\left(\mu^{*} \sigma\right)$ is the constant magnetic diffusivity or viscosity. For infinitely conducting fluids, $\nu_{H} \rightarrow 0$ and the diffusion equation is modified to

$$
u \frac{\partial H}{\partial x}+v \frac{\partial H}{\partial y}=0
$$

Plane constantly-inclined flows. The magnetic lines and the streamlines for these flows are constantly-inclined at a nonzero angle so that the angle between $\mathbf{V}$ and $\mathbf{H}$ is denoted by $\phi=$ constant $\neq 0$ everywhere. We study infinitely conducting flows only. Using Eq. (3) and the scalar product of $\mathbf{V}$ and $\mathbf{H}$, we obtain

$$
\begin{gathered}
u H_{2}-v H_{1}=q H \sin \phi=A, \\
u H_{1}+v H_{2}=q H \cos \phi=A \cot \phi,
\end{gathered}
$$

where $q=\sqrt{u^{2}+v^{2}}$ is the speed, $H=\sqrt{H_{1}^{2}+H_{2}^{2}}$ is the magnetic intensity magnitude, and $A \neq 0$ is an arbitrary constant. Considering these equations as two linear algebraic equations in the unknowns $H_{1}$ and $H_{2}$, we solve in terms of $u$ and $v$ to obtain

$$
H_{1}=A\left[\frac{u \cot \phi-v}{u^{2}+v^{2}}\right], \quad H_{2}=A\left[\frac{v \cot \phi+u}{u^{2}+v^{2}}\right] \text {. }
$$

Employing (16) in (1), (2), and (4), plane constantly-inclined infinitely conducting flows are governed by the system of five equations:

$$
\begin{gathered}
\frac{\partial u}{\partial x}+\frac{\partial v}{\partial y}=0 \quad \text { (continuity), } \\
\left(u \frac{\partial u}{\partial x}+v \frac{\partial u}{\partial y}\right)+\frac{\partial p}{\partial x} \\
=\mu \nabla^{2} u-\mu^{*} A j\left[\frac{u+v \cot \phi}{u^{2}+v^{2}}\right] \quad \text { (linear momentum), } \\
\rho\left(u \frac{\partial v}{\partial x}+v \frac{\partial v}{\partial y}\right)+\frac{\partial p}{\partial y}=\mu \nabla^{2} v+\mu^{*} A j\left[\frac{-v+u \cot \phi}{u^{2}+v^{2}}\right], \\
\left(2 u v-2 u^{2} \cot \phi\right) \frac{\partial u}{\partial x}+\left(v^{2}-u^{2}-2 u v \cot \phi\right) \frac{\partial u}{\partial y} \\
+\left(v^{2}-u^{2}-2 u v \cot \phi\right) \frac{\partial v}{\partial x}-\left(2 u v+2 v^{2} \cot \phi\right) \frac{\partial v}{\partial y}=0 \quad \text { (solenoidal), }
\end{gathered}
$$




$$
\frac{\partial}{\partial x}\left[\frac{u+v \cot \phi}{u^{2}+v^{2}}\right]-\frac{\partial}{\partial y}\left[\frac{u \cot \phi-v}{u^{2}+v^{2}}\right]=\frac{j}{A} \quad \text { (current density) }
$$

in four unknowns $u(x, y), v(x, y), p(x, y)$, and $j(x, y)$.

\section{Compatibility equations.}

Infinitely conducting aligned flows. For these flows Eq. (8), obtained from Eq. (3), is identically satisfied and the flow is governed by a system of six equations in six unknown functions $u, v, p, f, H_{1}$, and $H_{2}$ as functions of $x, y$.

A given velocity vector field $\mathbf{V}$ and a scalar function $f$ are compatible with the linear momentum equations (6) and (7) if these equations can be integrated to give the pressure function. An equation obtained by using the integrability condition $\partial^{2} p / \partial x \partial y=\partial^{2} p / \partial y \partial x$ is called the compatibility equation.

If we take $\psi=\psi(x, y)$ to be the streamfunction such that

$$
\frac{\partial \psi}{\partial x}=-v, \quad \frac{\partial \psi}{\partial y}=u,
$$

then Eq. (5) is identically satisfied.

Using (22) in the compatibility equation obtained from Eqs. (6) and (7), and using (22) in (9), we find that $\psi(x, y)$ and $f(x, y)$ must satisfy

$$
\left(\rho-\mu^{*} f^{2}\right) \frac{\partial\left(\psi, \nabla^{2} \psi\right)}{\partial(x, y)}+\mu \nabla^{4} \psi+\mu^{*} f \frac{\partial\left(|\nabla \psi|^{2}, f\right)}{\partial(x, y)}=0
$$

and

$$
\frac{\partial(f, \psi)}{\partial(x, y)}=0
$$

Finitely conducting aligned flows. The flow is governed by Eqs. (5)-(10) as Eq. (8) is not identically satisfied in this case. Proceeding as in the case of infinitely conducting flows, $f(x, y)$ and $\psi(x, y)$ must satisfy equations $(23),(24)$, and

$$
f \nabla^{2} \psi+\frac{\partial \psi}{\partial x} \frac{\partial f}{\partial x}+\frac{\partial \psi}{\partial y} \frac{\partial f}{\partial y}+k=0
$$

Employing Eqs. (24) and (25), Eq. (23) can be simplified as

$$
\rho \frac{\partial\left(\psi, \nabla^{2} \psi\right)}{\partial(x, y)}+\mu \nabla^{4} \psi=0
$$

so that the functions $f(x, y)$ and $\psi(x, y)$ must satisfy (24), (25), and (26).

Having obtained the functions $f(x, y)$ and $\psi(x, y)$ for finitely or infinitely conducting aligned MHD flows, we obtain the other flow variables by using Eqs. (22), (6), (7), and (10).

Finitely and infinitely conducting transverse flows. Introducing the function $\psi=$ $\psi(x, y)$, such that (22) holds true, in the system of four equations (11)-(14) governing the transverse flows and employing the integrability condition for pressure by using the linear momentum equations, we note that (11) is identically satisfied and 
we obtain a system of two equations

$$
\begin{gathered}
\rho \frac{\partial\left(\psi, \nabla^{2} \psi\right)}{\partial(x, y)}+\mu \nabla^{4} \psi=0, \\
\frac{\partial(\psi, H)}{\partial(x, y)}=\nu_{H}\left(\frac{\partial^{2} H}{\partial x^{2}}+\frac{\partial^{2} H}{\partial y^{2}}\right)
\end{gathered}
$$

in two unknown functions $\psi(x, y)$ and $H(x, y)$ for a finitely conducting transverse MHD fluid flow. The system of equations (27) and (28), with the right-hand side of (28) equal to zero, gives us the system of two equations in two unknowns for the infinitely conducting transverse MHD flows. Having solved this system, the other flow variables are given by Eqs. (22), (12), and (13).

Infinitely conducting constantly-inclined flows. Introducing the function $\psi=$ $\psi(x, y)$, such that (22) holds true, in the system of five equations (17)-(21) governing the constantly inclined flows and employing the integrability condition for pressure by using the linear momentum equations, we note that (17) is identically satisfied and the function $\psi(x, y)$ must satisfy the two equations

$$
\begin{aligned}
& |\nabla \psi|^{2}\left\{\rho \frac{\partial\left(\psi, \nabla^{2} \psi\right)}{\partial(x, y)}+\mu \nabla^{4} \psi\right\} \\
& +\mu^{*} A\left\{\cot \phi \frac{\partial(\psi, j)}{\partial(x, y)}-\left(\frac{\partial \psi}{\partial x} \frac{\partial j}{\partial x}+\frac{\partial \psi}{\partial y} \frac{\partial j}{\partial y}\right)\right\}=0 \\
& \left\{\left(\frac{\partial \psi}{\partial x}\right)^{2}-\left(\frac{\partial \psi}{\partial y}\right)^{2}+2 \frac{\partial \psi}{\partial x} \frac{\partial \psi}{\partial y} \cot \phi\right\}\left(\frac{\partial^{2} \psi}{\partial y^{2}}-\frac{\partial^{2} \psi}{\partial x^{2}}\right) \\
& +2\left\{\left[\left(\frac{\partial \psi}{\partial x}\right)^{2}-\left(\frac{\partial \psi}{\partial y}\right)^{2}\right] \cot \phi-2 \frac{\partial \psi}{\partial x} \frac{\partial \psi}{\partial y}\right\} \frac{\partial^{2} \psi}{\partial x \partial y}=0
\end{aligned}
$$

where

$$
j=A\left[\frac{\partial}{\partial x}\left\{\frac{1}{|\nabla \psi|^{2}}\left(\frac{\partial \psi}{\partial y}-\cot \phi \frac{\partial \psi}{\partial x}\right)\right\}+\frac{\partial}{\partial y}\left\{\frac{1}{|\nabla \psi|^{2}}\left(\frac{\partial \psi}{\partial x}-\cot \phi \frac{\partial \psi}{\partial y}\right)\right\}\right] .
$$

Having obtained $\psi(x, y)$ which satisfies (29) and (30), the flow variables are obtained by using Eqs. (22), (16), (18), and (19).

4. Riabouchinsky-type problems for aligned flows. Considering infinitely conducting aligned flows, we follow Ratip Berker's [1] approach and assume that

$$
\psi(x, y)=y F(x)+G(x) \text {. }
$$

Substituting (31) in Eqs. (23) and (24), we obtain the two equations

$$
\begin{aligned}
\left(\rho-\mu^{*} f^{2}\right)\left\{\left[y F^{\prime}(x)+G^{\prime}(x)\right] F^{\prime \prime}(x)-\right. & {\left.\left[y F^{\prime \prime \prime}(x)+G^{\prime \prime \prime}(x)\right] F(x)\right\} } \\
& +\mu\left[y F^{(i v)}(x)+G^{(i v)}(x)\right] \\
& +\mu^{*} f\left[\left\{2\left[y F^{\prime}(x)+G^{\prime}(x)\right]\left[y F^{\prime \prime}(x)+G^{\prime \prime}(x)\right]+2 F(x) F^{\prime}(x)\right\} \frac{\partial f}{\partial y}\right. \\
& \left.-2 F^{\prime}(x)\left[y F^{\prime}(x)+G^{\prime}(x)\right] \frac{\partial f}{\partial x}\right]=0
\end{aligned}
$$


and

$$
f=f(\psi),
$$

where the prime denotes differentiation with respect to $x$. Equation (32) is a fourthorder nonlinear differential equation with variable coefficients involving three arbitrary functions $F(x), G(x)$, and $f(\psi)$. Since by assuming a priori a functional form for the proportionality function $f(\psi)$ one may obtain a solution set for the flow problem, we derive various solution sets for our flow problem by investigating the following two forms for the function $f(\psi)$ :

I. $f(\psi)=a ; a \neq 0$ is any constant.

II. $f(\psi)=\psi=y F(x)+G(x)$.

We now study these two forms separately.

Form I $(f(\psi)=a)$. Taking $f(\psi)=a, a \neq 0$, in Eq. (32), we obtain

$$
\begin{aligned}
& \left(\rho-\mu^{*} a^{2}\right)\left\{\left[y F^{\prime}(x)+G^{\prime}(x)\right] F^{\prime \prime}(x)-\left[y F^{\prime \prime \prime}(x)+G^{\prime \prime \prime}(x)\right] F(x)\right\} \\
& \quad+\mu\left[y F^{(i v)}(x)+G^{(i v)}(x)\right]=0 .
\end{aligned}
$$

Since $x$ and $y$ are two independent variables and this equation must hold true for all values of $y$, it follows that the coefficients of different powers of $y$ must be zero so that the unknown functions $F(x)$ and $G(x)$ must satisfy

$$
\begin{aligned}
& \left(\rho-\mu^{*} a^{2}\right)\left[F^{\prime}(x) F^{\prime \prime}(x)-F(x) F^{\prime \prime \prime}(x)\right]+\mu F^{(i v)}(x)=0, \\
& \left(\rho-\mu^{*} \alpha^{2}\right)\left[F^{\prime \prime}(x) G^{\prime}(x)-F(x) G^{\prime \prime \prime}(x)\right]+\mu G^{(i v)}(x)=0 .
\end{aligned}
$$

Employing (22) and (31) in (6) and (7) and making use of (34) and (35), we find that the pressure distribution is given by

$$
\begin{aligned}
p= & \mu F^{\prime}(x)-\frac{1}{2} y^{2}\left[A+\mu^{*} a^{2} F^{\prime 2}(x)\right]-y\left[B+\mu^{*} a^{2} F^{\prime}(x) G^{\prime}(x)\right] \\
& -\frac{1}{2} \mu^{*} a^{2} G^{\prime 2}(x)-\frac{1}{2} \rho F^{2}(x)+p_{0},
\end{aligned}
$$

where $A, B$, and $p_{0}$ are arbitrary constants.

Equation (34) admits the following three particular solutions:

$$
\begin{aligned}
& F(x)=F_{1}(x)=-\frac{6 \mu}{\left(\rho-\mu^{*} a^{2}\right) x}, \\
& F(x)=F_{2}(x)=\frac{\mu b}{\rho-\mu^{*} a^{2}}\left(1+k e^{b x}\right), \\
& F(x)=F_{3}(x)=D x+E,
\end{aligned}
$$

where $b, k, D$, and $E$ are arbitrary constants.

Taking $F(x)=F_{1}(x)$, Eq. (35) becomes a Cauchy-Euler differential equation which upon integration yields $G(x)=a_{1} x^{3}+a_{2} / x+a_{3} / x^{2}+a_{4}$ so that

$$
\psi(x, y)=y F_{1}(x)+G(x)=-\frac{6 \mu}{\rho-\mu^{*} a^{2}} \frac{y}{x}+a_{1} x^{3}+\frac{a_{2}}{x}+\frac{a_{3}}{x^{2}}+a_{4},
$$

where $a_{1}, a_{2}, a_{3}$, and $a_{4}$ are arbitrary constants. 
Substituting $F(x)=F_{2}(x)$, Eq. (35) takes the form

$$
\begin{gathered}
R^{\prime \prime}(x)+b\left(2-k e^{b x}\right) R^{\prime}(x)+b^{2}\left(1-2 k e^{b x}\right) R=0 \\
R(x)=\frac{d}{d x}\left[G^{\prime}(x) e^{-b x}\right] .
\end{gathered}
$$

Multiplying this equation by $e^{b x}$ and integrating twice with respect to $x$, we obtain

$$
R(x)=a_{5} \exp \left[k e^{b x}-b x\right] \int \exp \left[-k e^{b x}\right] d x+a_{6} \exp \left[k e^{b x}-b x\right],
$$

where $a_{5}$ and $a_{6}$ are arbitrary constants. We therefore have

$$
\begin{aligned}
\psi(x, y)= & y F_{2}(x)+G(x) \\
= & \frac{\mu b}{\rho-\mu^{*} a^{2}}\left(1+k e^{b x}\right) y \\
& +a_{5} \int e^{b x}\left\{\int \exp \left(k e^{b x}-b x\right)\left[\int \exp \left(-k e^{b x}\right) d x\right] d x\right\} d x \\
& +a_{6} \int e^{b x}\left\{\int \exp \left(k e^{b x}-b x\right) d x\right\} d x+a_{7} e^{b x}+a_{8},
\end{aligned}
$$

where $a_{7}$ and $a_{8}$ are arbitrary constants.

Using $F(x)=F_{3}(x)$ in Eq. (35), solving the resulting fourth-order linear differential equation, and employing the expressions for $F_{3}(x)$ and $G(x)$ in (31), we have

$$
\begin{aligned}
& \psi(x, y)=y F_{3}(x)+G(x)=y(D x+E) \\
& +\iiint b_{1} \exp \left[\frac{\rho-\mu^{*} a^{2}}{\mu}\left(\frac{D}{2} x^{2}+E x\right)\right] d x d x d x+b_{2} x^{2}+b_{3} x+b_{4},
\end{aligned}
$$

where the $b_{i}, i=1,2,3,4$, are arbitrary constants.

Having obtained the streamfunction $\psi(x, y)$ for the three particular cases given by Eqs. (37), (38), and (39), the pressure for each of these three particular cases is found by substituting the corresponding $F(x)$ and $G(x)$ in Eq. (36).

We now consider the special case of (31) when $G=0$, which is usually attributed to Riabouchinsky [3]. Physically such a flow represents a plane stagnation flow in which the flow is separated in the two symmetrical regions by the plane $X=0$. For this special case, Eqs. (37), (38), and (39) are respectively replaced by

$$
\psi(x, y)=-\frac{6 \mu}{\rho-\mu^{*} a^{2}} \frac{y}{x}, \quad \psi(x, y)=\frac{\mu b}{\rho-\mu^{*} a^{2}}\left(1+k e^{b x}\right) y,
$$

and $\psi(x, y)=(D x+E) y$.

Form II $(f(x)=\psi=y F(x)+G(x))$. Using $f(x)=y F(x)+G(x)$ in Eq. (32), we obtain a cubic equation in $y$, with coefficients as functions of $x$, to hold true. The coefficients of this cubic equation vanish simultaneously and, therefore, $F(x)$ 
and $G(x)$ must satisfy

$$
\begin{gathered}
\rho\left[F^{\prime \prime}(x) G^{\prime}(x)-F(x) G^{\prime \prime \prime}(x)\right]+\mu G^{(i v)}(x) \\
+\mu^{*}\left[F(x) G^{2}(x) G^{\prime \prime \prime}(x)-F^{\prime \prime}(x) G^{2}(x) G^{\prime}(x)\right. \\
-2 F^{\prime}(x) G(x) G^{2}(x)+2 F^{2}(x) G(x) F^{\prime}(x) \\
\left.+2 F(x) G(x) G^{\prime}(x) G^{\prime \prime}(x)\right]=0, \\
\rho\left[F^{\prime}(x) F^{\prime \prime}(x)-F(x) F^{\prime \prime \prime}(x)\right]+\mu F^{(i v)}(x) \\
+\mu^{*}\left[-F^{\prime}(x) F^{\prime \prime}(x) G^{2}(x)+F(x) F^{\prime \prime}(x) G^{2}(x)\right. \\
+2 F^{2}(x) G^{\prime}(x) G^{\prime \prime \prime}(x)-4 F^{\prime 2}(x) G(x) G^{\prime}(x) \\
-2 F(x) F^{\prime}(x) G^{\prime 2}(x)+2 F^{2}(x) F^{\prime}(x) \\
\left.+2 F(x) F^{\prime}(x) G(x) G^{\prime \prime}(x)+2 F^{2}(x) G^{\prime}(x) G^{\prime \prime}(x)\right]=0, \\
2 F^{2}(x) F^{\prime \prime \prime}(x) G(x)-F^{2}(x) F^{\prime \prime}(x) G^{\prime}(x)+F^{3}(x) G^{\prime \prime \prime}(x) \\
-2 F^{\prime 3}(x) G(x)-4 F(x) F^{\prime 2}(x) G^{\prime}(x)+2 F^{2}(x) F^{\prime}(x) G^{\prime \prime}(x)=0, \\
F^{2}(x) F^{\prime}(x) F^{\prime \prime}(x)+F^{3}(x) F^{\prime \prime \prime}(x)-2 F(x) F^{\prime 3}(x)=0 .
\end{gathered}
$$

Equation (43) admits the following three particular solutions:

$$
F(x)=F_{1}(x)=k e^{b x}, \quad F(x)=F_{2}(x)=D \sqrt{x}, \quad F(x)=F_{3}(x)=A,
$$

where $k, b, D$, and $A$ are arbitrary constants.

Taking $F(x)=F_{1}(x)$ in Eqs. (40), (41), and (42), we find that $k=0$ and $G(x)=0$. Using $F(x)=F_{2}(x)$ in Eqs. (40)-(42), we get $D=0$ and $G(x)=0$. Employing $F(x)=F_{3}(x)=A$ in Eqs. (40), (41), and (42), we obtain

$$
G(x)=B x+C,
$$

where $B$ and $C$ are arbitrary constants. Thus, a streamfunction is given by

$$
\psi(x, y)=A y+B x+C .
$$

We now consider finitely conducting fluid flows. Substituting Eqs. (22) and (31) in (25) and (26), we find that the functions $F(x), G(x)$, and $f(\psi)$ must satisfy

$$
\begin{gathered}
\mu F^{(i v)}(x)+\rho\left[F^{\prime}(x) F^{\prime \prime}(x)-F(x) F^{\prime \prime \prime}(x)\right]=0, \\
\mu G^{(i v)}(x)+\rho\left[F^{\prime \prime}(x) G^{\prime}(x)-F(x) G^{\prime \prime \prime}(x)\right]=0, \\
f\left[y F^{\prime \prime}(x)+G^{\prime \prime}(x)\right]+\left[y F^{\prime}(x)+G^{\prime}(x)\right] \frac{\partial f}{\partial x}+F(x) \frac{\partial f}{\partial y}=-k .
\end{gathered}
$$


Equations (44) and (45) are Eqs. (34) and (35) with $a=0$. Therefore, solutions of (44) and (45) are the same as those of (34) and (35) with $a=0$. Having obtained $F(x)$ and $G(x)$, we can find $f(x, y)$ by solving Eq. (46).

5. Riabouchinsky-type problems for transverse flows. Proceeding as in Sec. 4 and assuming that $\psi$ is given by (31), we employ (31) in (27) to find that $F(x)$ and $G(x)$ must satisfy

$$
\begin{gathered}
\mu F^{(i v)}(x)+\rho\left[F^{\prime}(x) F^{\prime \prime}(x)-F(x) F^{\prime \prime \prime}(x)\right]=0, \\
\mu G^{(i v)}(x)+\rho\left[F^{\prime \prime}(x) G^{\prime}(x)-F(x) G^{\prime \prime \prime}(x)\right]=0 .
\end{gathered}
$$

Employing (22) and (31) in (12) and (13) and making use of (47) and (48), we find that the pressure distribution is

$$
p(x, y)=\mu F^{\prime}(x)-\frac{1}{2} \rho F^{2}(x)-\frac{1}{2} A y^{2}-B y-\frac{1}{2} \mu^{*} H^{2}+p_{0},
$$

where $A, B$, and $p_{0}$ are arbitrary constants.

Equation (47) admits the following three particular solutions:

$$
\begin{gathered}
F(x)=F_{1}(x)=-\frac{6 \mu}{\rho x}, \quad F(x)=F_{2}(x)=\frac{\mu a}{\rho}\left(1+k e^{a x}\right), \\
F(x)=F_{3}(x)=D x+E,
\end{gathered}
$$

where $a, k, D$, and $E$ are arbitrary constants.

Following the same procedure as in Form I of Sec. 4, we find that

(i) When $F(x)=F_{1}(x)$, the streamfunction is given by

$$
\psi(x, y)=-\frac{6 \mu}{\rho} \frac{y}{x}+a_{1} x^{3}+\frac{a_{2}}{x}+\frac{a_{3}}{x^{2}}+a_{4},
$$

where $a_{1}, a_{2}, a_{3}$, and $a_{4}$ are arbitrary constants. The streamline patterns are shown in Figs. 1 and 2 (see pp. 282 and 283). 


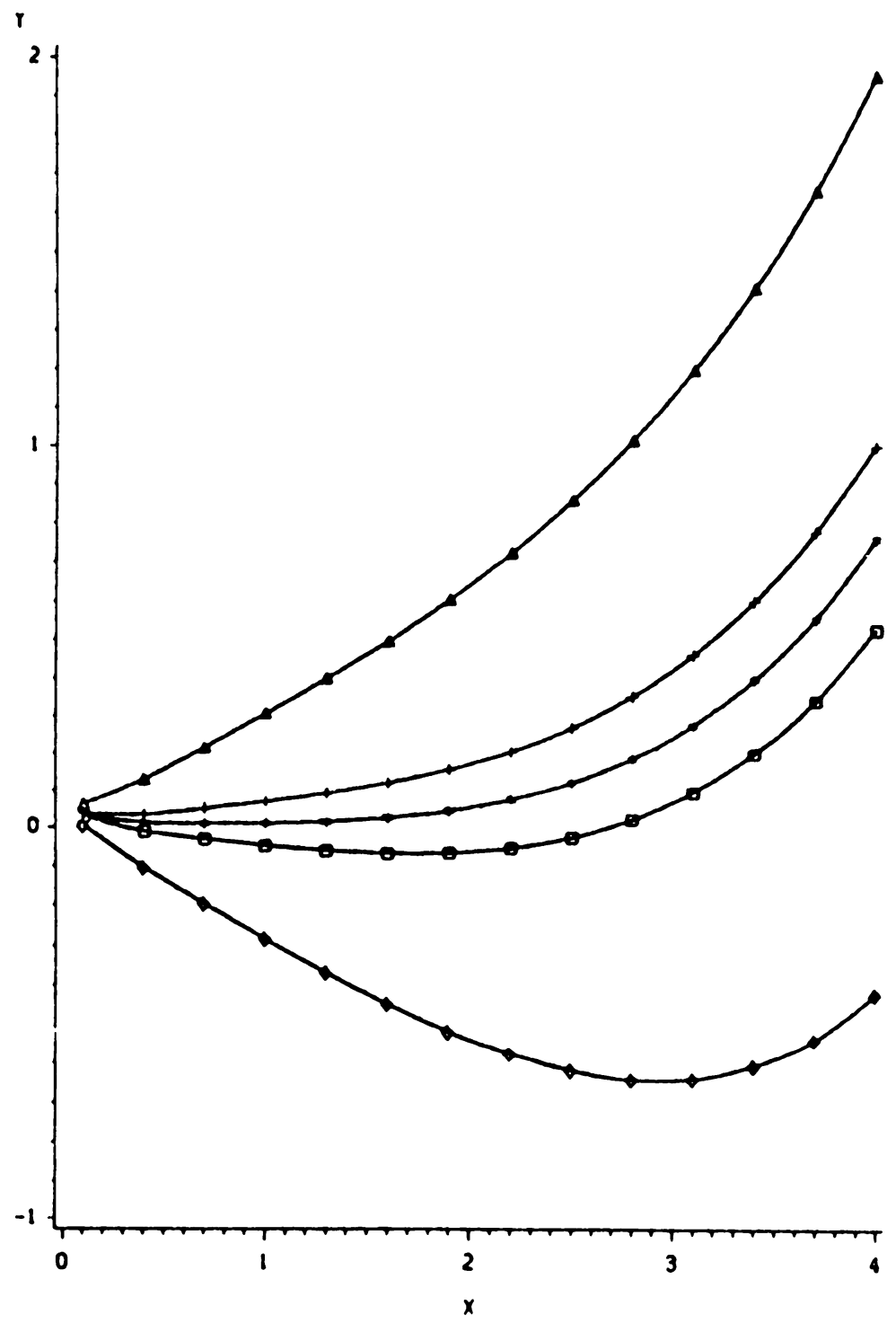

FIG. 1. Streamline pattern of Eq. (49) with $\mu / \rho=0.0178, a_{1}=a_{2}=$ $a_{3}=1, a_{4}=0$. 


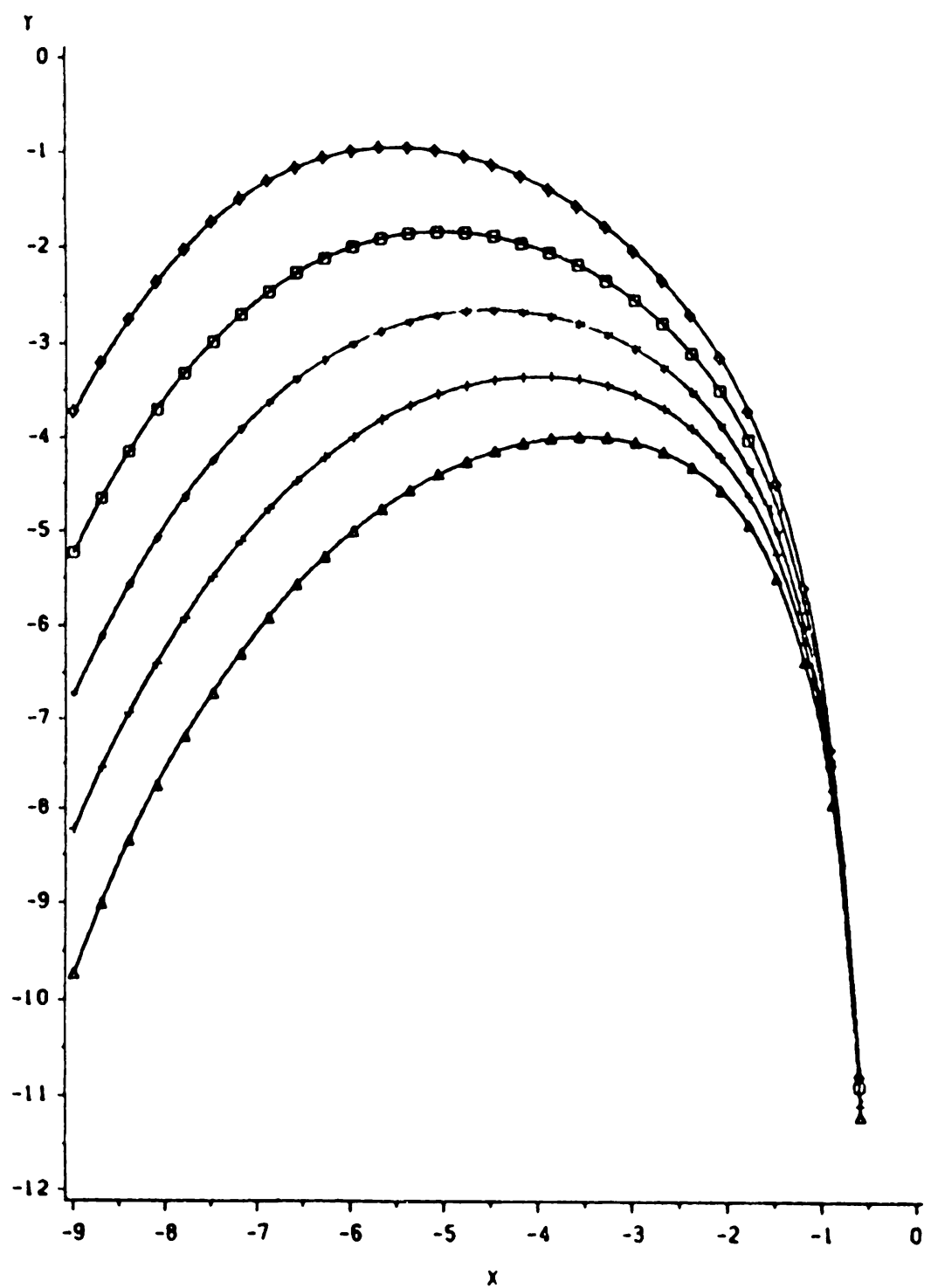

FIG. 2. Streamline pattern of Eq. (49) with $\mu / \rho=1, a_{1}=1 / 216$, $a_{2}=-36, a_{3}=6, a_{4}=0$.

(ii) When $F(x)=F_{2}(x)$, the streamfunction takes the form

$$
\begin{aligned}
\psi(x, y)= & \frac{\mu a}{\rho}\left(1+k e^{a x}\right) y \\
& +a_{5} \int e^{a x}\left\{\int \exp \left(k e^{a x}-a x\right)\left[\int \exp \left(-k e^{a x}\right) d x\right]\right\} d x \\
& +a_{6} \int e^{a x}\left\{\int \exp \left(k e^{a x}-a x\right) d x\right\} d x+a_{7} e^{a x}+a_{8},
\end{aligned}
$$

where the $a_{i}, i=5,6,7,8$, are arbitrary constants. In the special case where 
$k=0$, the streamfunction is given by

$$
\psi(x, y)=\frac{\mu}{\rho} a y+a_{9} e^{a x}+a_{10} x^{2}+a_{11} x+a_{12},
$$

where $a_{9}, \ldots, a_{12}$ are arbitrary constants. The streamline pattern is shown in Fig. 3.

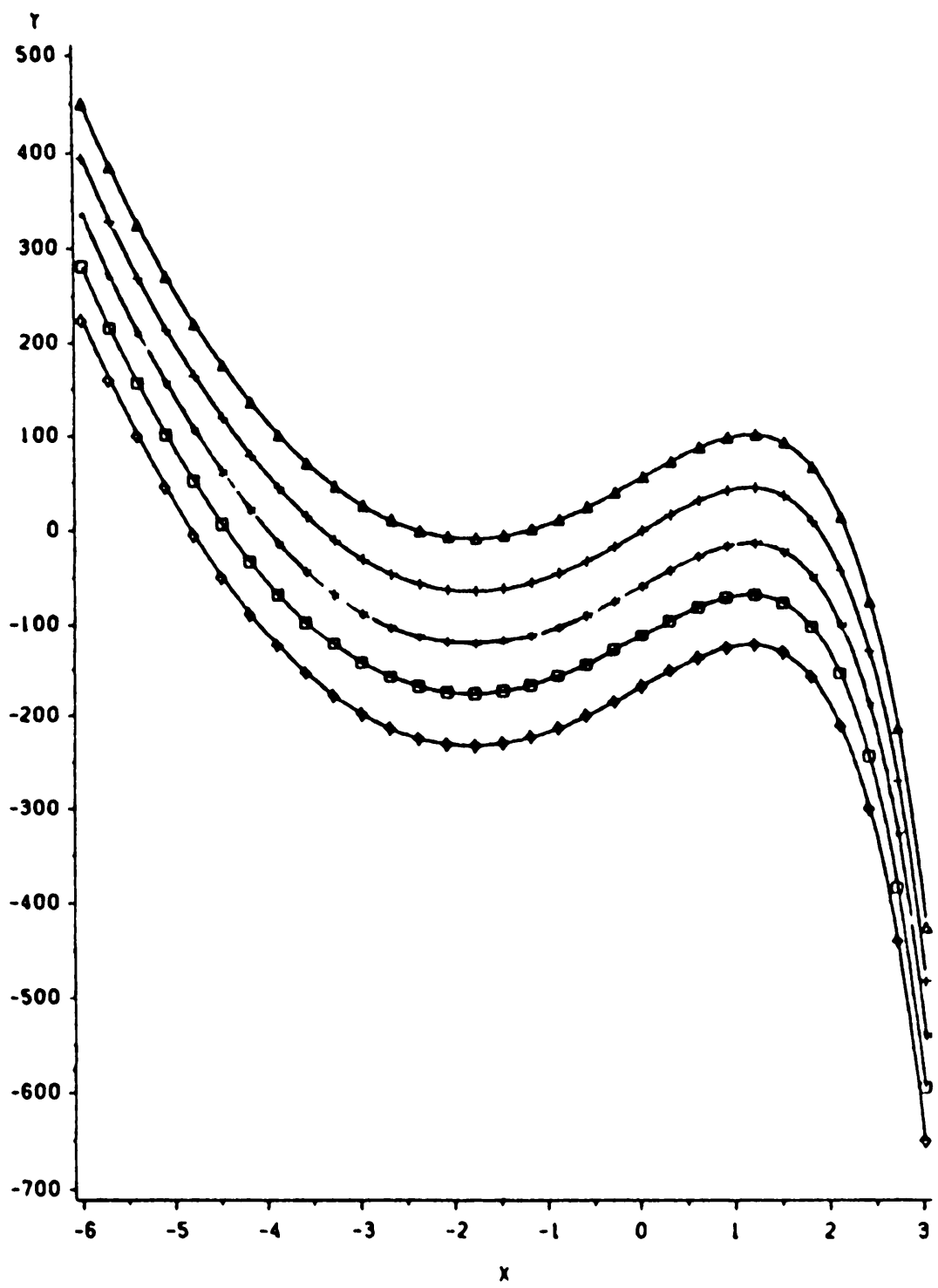

FIG. 3. Streamline pattern of Eq. (50) with $\mu / \rho=1, a_{2}=a_{9}=1$, $a_{10}=-0.5, a_{11}=-2, a_{12}=0$. 
RIABOUCHINSKY FLOWS IN MAGNETOHYDRODYNAMICS

285

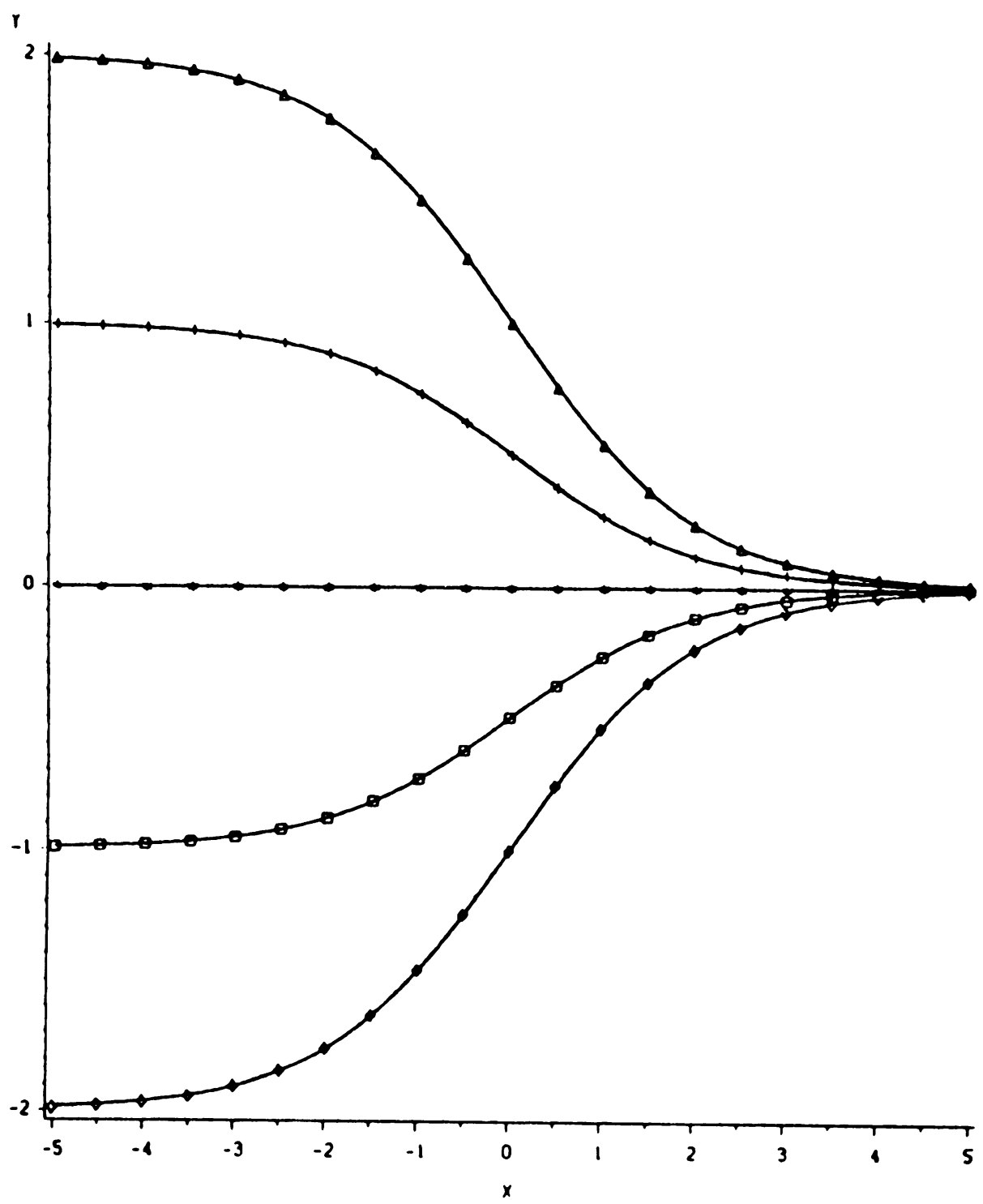

FIG. 4. Streamline pattern of Eq. (51) with $\mu / \rho=1, a=k=1$. 


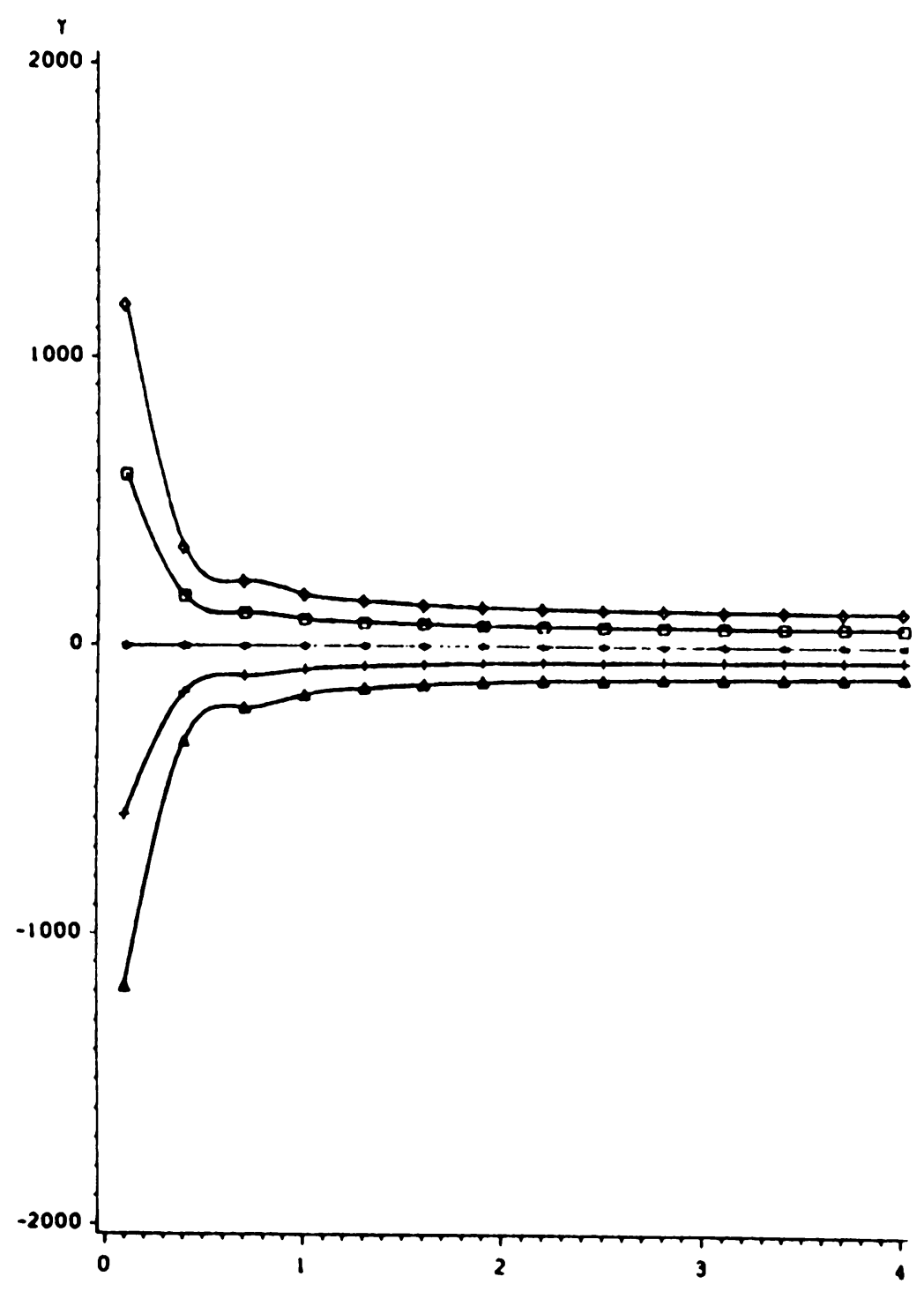

FIG. 5. Streamline pattern of Eq. (51) with $\mu / \rho=0.0178, a_{1}=k=-1$.

In the particular case of $(31)$ when $G(x)=0$, we find that the streamfunction is

$$
\psi(x, y)=\frac{\mu a}{\rho}\left(1+k e^{a x}\right) y .
$$

The streamlines patterns are shown in Figs. 4 and 5.

(iii) When $F=F_{3}(x)$, the streamfunction takes the form $\psi(x, y)=(D x+E) y+b_{1} \iiint \exp \left[\frac{\rho}{\mu}\left(\frac{D}{2} x^{2}+E x\right)\right] d x d x d x+b_{2} x^{2}+b_{3} x+b_{4}$,

where $b_{1}, b_{2}, b_{3}$, and $b_{4}$ are arbitrary constants. 
6. Riabouchinsky-type problems for constantly-inclined flows. Assuming that $\psi$ is given by Eq. (31), we employ (31) in (30) to find that $F(x)$ and $G(x)$ must satisfy

$$
\begin{gathered}
{\left[F^{2}(x) G^{\prime \prime}(x)-4 F(x) F^{\prime}(x) G^{\prime}(x)-G^{\prime 2}(x) G^{\prime \prime}(x)\right]} \\
+\cot \phi\left[2 F^{\prime}(x) G^{2}(x)-2 F^{2}(x) F^{\prime}(x)-2 F(x) G^{\prime}(x) G^{\prime \prime}(x)\right]=0 \\
{\left[F^{2}(x) F^{\prime \prime}(x)-4 F(x) F^{\prime 2}(x)-2 F^{\prime}(x) G^{\prime}(x) G^{\prime \prime}(x)-F^{\prime \prime}(x) G^{\prime 2}(x)\right]} \\
+\cot \phi\left[4 F^{\prime 2}(x) G^{\prime}(x)-2 F(x) F^{\prime}(x) G^{\prime \prime}(x)-2 F(x) F^{\prime \prime}(x) G^{\prime}(x)\right]=0 \\
{\left[F^{\prime 2}(x) G^{\prime \prime}(x)+2 F^{\prime}(x) F^{\prime \prime}(x) G^{\prime}(x)\right]} \\
+\cot \phi\left[2 F(x) F^{\prime}(x) F^{\prime \prime}(x)-2 F^{\prime 3}(x)\right]=0 \\
F^{\prime 2}(x) F^{\prime \prime}(x)=0 .
\end{gathered}
$$

Equation (55) implies that either $F^{\prime \prime}(x)=0$ with $F^{\prime}(x) \neq 0$ or $F^{\prime}(x)=0$. We study these two cases separately.

Case I $\left(F^{\prime \prime}=0\right.$ and $\left.F^{\prime} \neq 0\right)$. Employing $F^{\prime \prime}(x)=0$ in Eqs. (53) and (54), we get

$$
G^{\prime \prime}(x)-2 F^{\prime}(x) \cot \phi=0
$$

and

$$
2 F^{\prime}(x)+G^{\prime \prime}(x) \cot \phi=0 .
$$

Eliminating $G^{\prime \prime}$ between these two equations, we get

$$
F^{\prime}(x)\left[1+\cot ^{2} \phi\right]=0 .
$$

This equation implies that $F^{\prime}(x)=0$ since $1+\cot ^{2} \phi \neq 0$ which contradicts our assumption that $F^{\prime}(x) \neq 0$.

Case II $\left(F^{\prime}(x)=0\right)$. Taking $F^{\prime}(x)=0$ or $F(x)=a$ in Eqs. (52), (53), and (54), where $a \neq 0$ is an arbitrary constant, we find that (53) and (54) are identically satisfied and (52) gives

$$
G^{\prime \prime}(x)\left[G^{\prime 2}(x)+2 a G^{\prime}(x) \cot \phi-a^{2}\right]=0 .
$$

Equation (56) implies that either $G^{\prime \prime}(x)=0$ or $G^{2}(x)+2 a G^{\prime}(x) \cot \phi-a^{2}=0$.

When $G^{\prime \prime}(x)=0$, the streamfunction takes the form

$$
\psi(x, y)=a y+b_{1} x+b_{2}
$$

and the pressure distribution is given by

$$
p(x, y)=p_{0}-\frac{1}{2} \rho\left(a^{2}+b_{1}^{2}\right),
$$

where $b_{1}, b_{2}$, and $p_{0}$ are arbitrary constants. The streamline pattern is shown in Fig. 6 . 


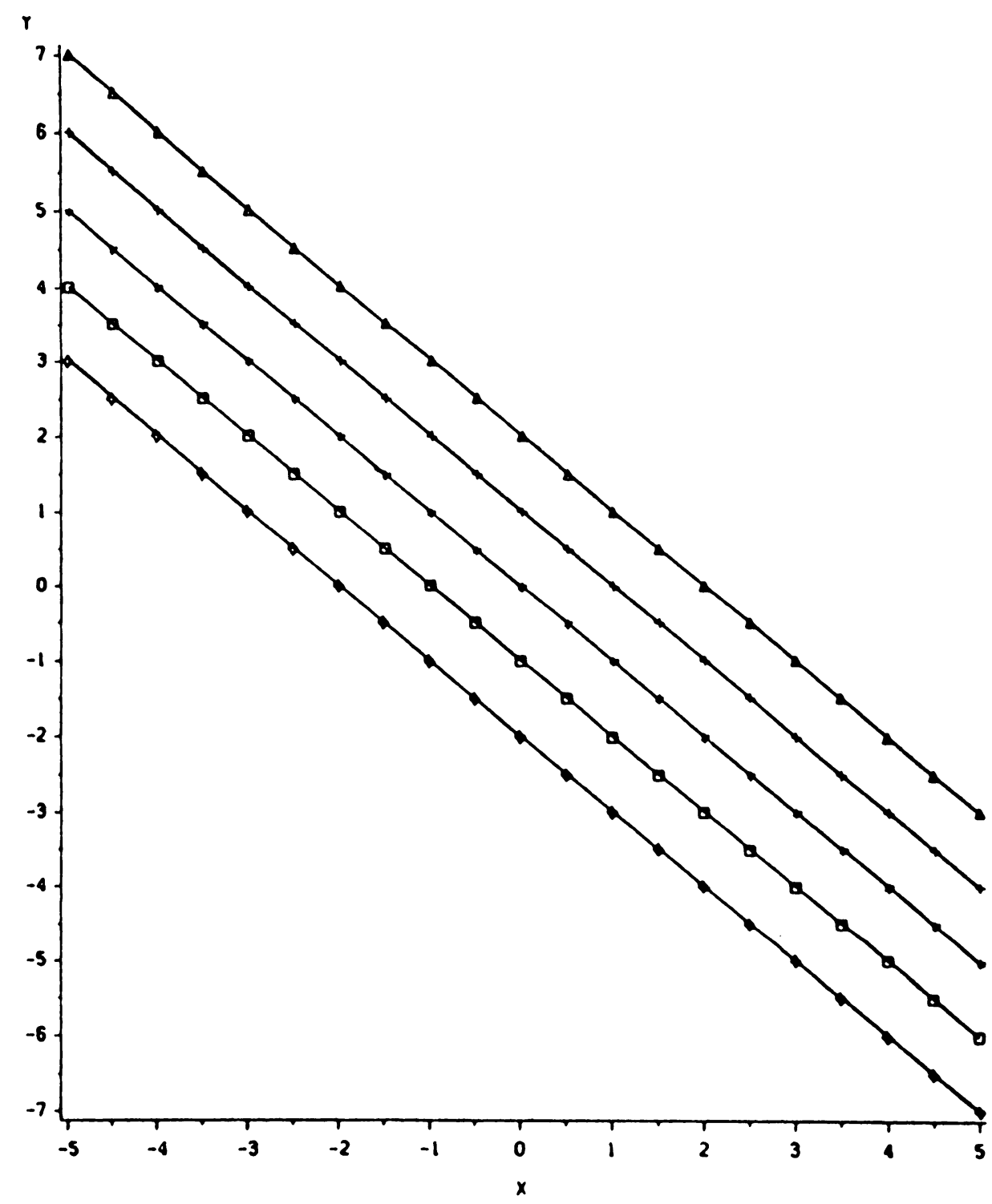

FIG. 6. Streamline pattern of Eq. (57) with $a=b_{1}=1, b_{2}=0$.

When $G^{\prime 2}(x)+2 a G^{\prime}(x) \cot \phi-a^{2}=0$, the streamfunction takes the form

$$
\psi(x, y)=a y+\left\{-a \cot \phi \pm a \sqrt{1+\cot ^{2} \phi}\right\} x+b_{3}
$$

and the pressure distribution is given by

$$
p=p_{0}-\frac{1}{2} \rho\left\{a^{2}+\left[-a \cot \phi \pm a \sqrt{1+\cot ^{2} \phi}\right]^{2}\right\}
$$

where $b_{3}$ and $p_{0}$ are arbitrary constants.

7. Conclusions and physical applications. Riabouchinsky [3] determined exact integrals for steady plane viscous incompressible fluid flow in 1924 by assuming a linear 
form for $\psi$ given in (31). These solutions of the flow equations did not provide flows with solid and fixed boundaries or with movable obstacles. The flow patterns of these exact integrals led Stuart [6], Tamada [7], and Dorrepaal [8] to study nonorthogonal stagnation point flow in two dimensions.

The present work determines exact integrals for viscous MHD flows when the magnetic field is aligned, transverse, or constantly-inclined. Various resulting flow patterns are drawn. It is hoped that physical problems such as orthogonal and oblique stagnation point flows will result from the obtained integrals.

Acknowledgment. The authors are thankful to the referee for valuable comments and suggestions. The work reported in this paper has been supported by Grant No. A7161 of N.S.E.R.C. of Canada.

\section{REFERENCES}

[1] R. Berker, Intégration des équations de mouvement d'un fluide visgueux incompressible, Vol. VIII/2, Stromungs Mechanik II, Handbuch der Physik, Berlin, 1963

[2] R. Berker, Sur les équations de compatibilité relatives an mouvement d'un Gaz, C. R. Acad. Sci. Paris, Sér. I Math. 242, 342-344 (1956)

[3] D. Riabouchinsky, Some considerations regarding plane irrotational motion of a liquid, C. R. Hebd. Seanc. Acad. Sci. Paris 179, 1133-1136 (1924)

[4] P. N. Kaloni and K. Huschilt, Semi-inverse solutions of a non-Newtonian fluid, Internat. J. NonLinear Mech. 19, 373-381 (1984)

[5] S. I. Pai, Magnetohydrodynamics and Plasma Physics, Prentice-Hall, Englewood Cliffs, NJ, 1962

[6] J. T. Stuart, J. Aero/Space Sci. 26, 124-125 (1959)

[7] K. Tamada, Two dimensional stagnation-point flow impinging obliquely on a plane wall, J. Phys. Soc. Japan 46, 310-311 (1979)

[8] J. M. Dorrepaal, An exact solution of the Navier-Stokes equation which describes non-orthogonal stagnation point flow in two dimensions, J. Fluid Mech. 163, 141-147 (1986) 